\title{
Influence of the clinical in postural balance in woman with knee osteoarthritis
}

\begin{abstract}
Objective: This study aimed to examine the association between the postural balance and clinical status of patients with Knee osteoarthritis (KOA) with and without a history of falls.

Method: 74 women with KOA between the age of 50 and 75 years were selected, separated in three groups (KOA subjects with and without history of falls and asymptomatic subjects for KOA), by examining the association between clinical data and postural balance.

Results: The results indicated that the subjects with KOA, regardless of history of falls, needed more time to acquire postural stability than the asymptomatic. The balance of these subjects is significantly affected by increased body mass, pain intensity, levels of KOA, and fear of falling and diminished physical activity and functional capacity.
\end{abstract}

Conclusion: In light of these findings, we concluded that the clinical of KOA, rather than the history of falls, is an important predictor of falls risk.

Keywords: osteoarthritis, postural balance, clinical and social aspects.
Volume 4 Issue 4 - 2019

\author{
Ana Carolina Silva de Souza Moreira,' \\ Fernando Luiz Cardoso, ${ }^{2}$ Giovana Zarpellon \\ Mazo, ${ }^{2}$ Gilmar Moraes Santos ${ }^{3}$ \\ 'PhD in Human Movement Sciences, Santa Catarina State \\ University (UDESC), Brazil \\ 2Professor of the Graduate Program in Human Movement \\ Sciences, Santa Catarina State University (UDESC), Brazil \\ ${ }^{3}$ Professor of the Graduate Program in Physiotherapy, Santa \\ Catarina State University (UDESC), Brazil
}

\author{
Correspondence: : Ana Carolina Silva de Souza \\ Moreira,Universidade do Estado de Santa Catarina, Centro da \\ Ciência da Saúde e do Esporte/CEFID, Rua Pascoal Simone, \\ 358-Florianópolis/SC, Brazil, Tel 5548984I I3345, \\ Email anakarolfisio@gmail.com
}

Received: July II, 2019 | Published: July 16, 2019

\section{Introduction}

In recent decades, osteoarthritis (OA) has become the most common cause of disability and frailty, affecting $30-58 \%$ of the population above the age of $45 . .^{1-4}$ The knee joint is the second joint most commonly affected by this disease. Knee osteoarthritis (KOA) presents significant clinical implications and affects approximately $30 \%$ of men and $33 \%$ of women between the ages of 60 and $69 . .^{2-4}$

KOA is characterized by the degeneration of the joint cartilage, with progressive loss of functional capacity due to increasing deficits in the proprioceptive and neuromuscular systems, thus limiting the performance of activities of daily living (ADL) and negatively affecting quality of life.$^{5-8}$ Individuals with KOA experience episodes of pain, restricted movement, muscle weakness, and decreased motor coordination ${ }^{3,5,7-10}$ as the disease gradually affects precision, agility, and reflexes and ultimately impairs postural balance. ${ }^{11,12}$

According to recent studies, ${ }^{6,11-15}$ decreased postural balance and functional capacity are associated with increased risk of falling in the elderly. Proper postural balance is essential during ADLs and it is a key factor to avoiding injuries due to falls. ${ }^{6,14}$ Current evidence shows that people with KOA are likely to suffer deterioration of postural balance. ${ }^{3,6,14,15}$ Furthermore, it has been shown that patients with $\mathrm{KOA}$ are at greater risk of falling because of a lack of adequate neuromuscular response to balance disturbances ${ }^{5,8,10}$ during the performance of functional tasks and because of greater fear of falling. ${ }^{6,14}$

Current literature $;^{5,16,17}$ shows an association between decreased balance and decreased muscle strength (quadriceps) and proprioception in patients with KOA; however, the origin of that decreased balance remains unclear. Tarigan et al. ${ }^{18}$ found a correlation between body mass index and KOA severity, but there is no evidence of association between center of pressure (COP) parameters and clinical parameters (pain, severity of osteoarthritis, and age).
The results of previous studies ${ }^{19,20}$ are conflicting and do not explain the association between KOA and clinical parameters (balance deficit, functional capacity, and pain) or the association between KOA and falling. Tuna and Balci ${ }^{19}$ found no significant relationship between radiological findings and the functional capacity of the knee and quadriceps and hamstring muscle strength. However, there is limited association between the clinical picture of patients with KOA and postural balance and history of falls. ${ }^{20}$ Furthermore, only a limited number of studies have reported an association between postural control and functional capacity in this group of patients. ${ }^{17}$

Studies ${ }^{21,22}$ have been published on the risk factors of falls in the elderly, but it is still unclear whether the level of functional capacity is an essential factor for the risk of falls in the elderly and even less clear in patients with KOA. Therefore, it becomes necessary to investigate the relationship between the level of functional capacity and history of falls in postural balance, as falling is the biggest predictor of disability, particularly in the elderly. Given these considerations, this study aimed to examine the association between the postural balance and clinical status of patients with KOA with and without a history of falls, controlled by a control group with asymptomatic subjects for KOA.

\section{Materials and methods}

\section{Description of study and ethics statement}

This is a descriptive cross-sectional and observational study with a non-random sampling approach. This study was approved by the Ethics Committee of Santa Catarina State University (protocol no. 068/2018), pursuant to National Health Council Resolution 466/2012.

\section{Subjects}

The subjects were referred by the Orthopedics Department of the University Hospital of Santa Catarina Federal University (UFSC) 
and by the Physical Therapy School Clinic of Santa Catarina State University (UDESC), Brazil. The diagnosis of KOA was confirmed by a medical specialist in orthopedics and traumatology, using the criteria of the American College of Rheumatology, and through bilateral anteroposterior radiographs of the knee in a standing position obtained in the three months preceding the study. The severity of alteration of the tibiofemoral joint was classified by the KellgrenLawrence Grading Scale, a radiological classification of KOA officially accepted by the World Health Organization (WHO) ${ }^{23}$

The study recruited 74 women between the ages of 50 and 75 , including 42 women with bilateral KOA in the tibiofemoral compartment. Of the total sample, 20 had a history of falls and were included in the KOA Group - Falls $\left(\mathrm{KOAG}^{\mathrm{F}}\right)$ and 22 had no history of falls and were included in the KOA Group - No Falls $\left(\mathrm{KOAG}^{\mathrm{NF}}\right)$. The remaining 32 women were asymptomatic for KOA and were included in the control group (CG). To be included in the KOAG, the subject had to present with bilateral idiopathic KOA and be able to walk without pause or assistance for at least six meters. The control group was composed of independent walkers without signs and/or symptoms of KOA, according to the clinical examination and confirmed with X-rays.

This study excluded subjects with the following: history of lower limb and/or spinal surgery in the last 12 months; musculoskeletal pain symptoms or disorders that prevented the maintenance of a standing position for the time needed during data collection (approximately 30 minutes); use of walking aids such as a cane, crutch, or walker; diabetes mellitus type I or II; previous treatment for balance; neurological disorders diagnosed in physical therapy assessment and/or clinical setting; cognitive impairment (Mini-Mental State Examination score less than 23); and disturbances in the vestibular and cerebellar systems as indicated by the Romberg test, finger-to- nose test, Trendelenburg Lurch test, and other clinical neurological examinations.

\section{Instrumentation}

The Visual Analogue Scale (VAS) was used to check the intensity of pain ranging from 0 (no pain) to 10 (worst possible pain). The Western Ontario and McMaster Universities Osteoarthritis Index (WOMAC) was used in the investigation of the functional capacity of the knee joint. The Mini-Mental State Examination (MMSM) was used to assess the presence of cognitive impairment. The MMSM score classifies cognitive deficit as follows: preserved cognitive functions (30-26 points); no abnormalities suggestive of deficit (2624 points); and indicative of cognitive deficit (23 points or less). ${ }^{24}$ The Falls Efficacy Scale-International-Brazil (FES-I-BRAZIL) version adapted and validated by $\operatorname{Camarg}^{25}$ was used to assess fear of falling. The Chattecx Dynamic Balance System (Chattanooga Group Inc., Hixon, TN, USA) was used to evaluate center of pressure (COP) in the mediolateral (ML) and anteroposterior (AP) directions. The system features a predetermined frequency of $100 \mathrm{~Hz}$, a $15 \mathrm{~Hz}$ filter, and a software program that converts the amplified analogue signals of the transducers into digital signals.

To analyze the behavior of the COP, stabilogram diffusion analysis (SDA) was used. According to Collins and De Luca, ${ }^{26}$ this method of analysis measures the signal behavior of the COP to describe the evolutionary properties of the fractal time series and estimate additional information on the nature of the postural control system. The stabilogram-diffusion curve of the COP has two regions, one after a short-term interval and the other after a long-term interval. These intervals are separated by a critical period after which the slope of the graph goes through significant changes. Four posturographic parameters are extracted from these regions (Figure 1):

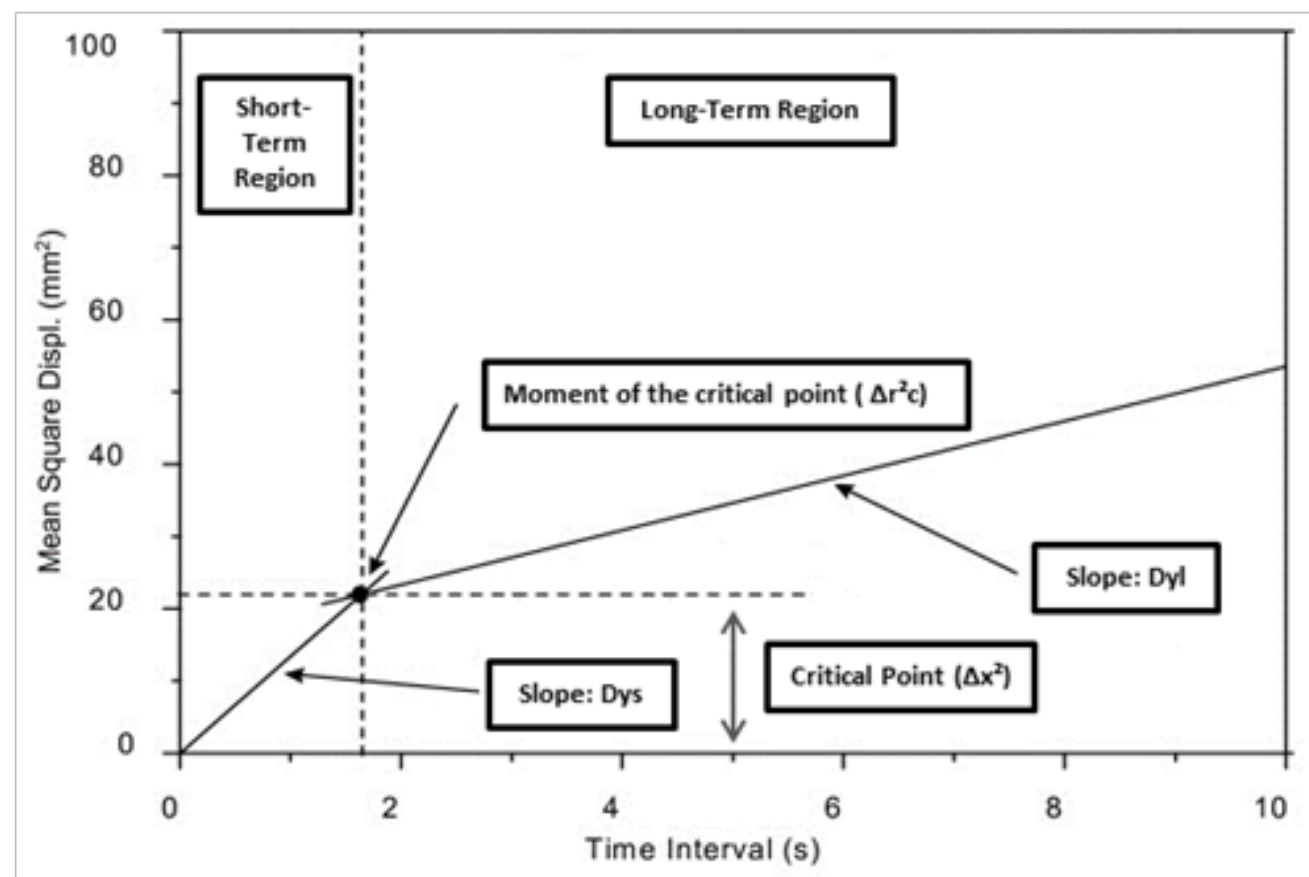

Figure I The figure illustrates stabilogram-diffusion curve of the COP, which is separated into two regions, which are Short-Term (Dys) and Long-Term and (Dyl) regions. Fitted line slopes in each region (Dys and Dyl) indicate the rate of change in magnitude of sway as a function of time-interval. The critical point $\left(\Delta x^{2}\right)$ and moment of the critical point $\left(\Delta r^{2} c\right)$ are the intersection of these two lines. 
Diffusion Coefficient: SDA allows the identification of this parameter in two regions of the graph, i.e., the short-term interval (Dys) and long-term interval (Dyl). These regions seem to imply the existence of a control scheme consisting of an open-loop (Dys) mechanism and a closed-loop (Dyl) mechanism to maintain postural equilibrium. ${ }^{26}$

Hurst exponent: Evaluates the probability of the COP moving away from or near a relative equilibrium, obtained by the slope of the linear regression of the stabilogram-diffusion graph, with values between $0.5<\mathrm{H}<1$ being associated with a persistent behavior. ${ }^{23}$

Critical point: $\left(\Delta \mathbf{x}^{2}\right)$ : Represents the position separating the two regions (short- and long-term intervals) in the stabilogram-diffusion graph. ${ }^{26}$

Moment of the critical point: $\left(\Delta \mathbf{r}^{2} \mathbf{c}\right)$ : Refers to the instant when the intersection of the two regions (short- and long-term intervals) occurred in the stabilogram-diffusion graph. ${ }^{26}$

\section{Procedures}

Initially, it was collected on their health status, age, race, marital status, disease, medications, dizziness, visual disorders, fear of falling, history of falls, and intensity of knee pain using the VAS. Because KOA is a chronic disease, some subjects reported constant use of analgesics and/or anti-inflammatory medications. As this was an observational and descriptive study and in order to portray the postural balance and functional mobility of everyday life, the subjects were not asked to discontinue their medication. The WOMAC questionnaire was administered in order to verify the level of functional capacity of the knee, and the MMSE questionnaire was administered to assess cognitive levels.

For the balance assessment, the subjects remained barefoot at rest on the Chattecx Balance System platform. They were instructed to maintain a standing posture with upper limbs parallel to the body, feet $10 \mathrm{~cm}$ apart not exceeding shoulder width, gaze directed forward, head straight, and without lateral tilt. Balance was measured on the platform in two conditions: with eyes open and closed. The data acquisition was 25 seconds long with a frequency of $100 \mathrm{~Hz}$ and pre-filtering of $15 \mathrm{~Hz}$ determined by the platform. There were three repetitions for each visual condition with a one-minute rest Table I Characteristics of subjects data presented as mean (SD) interval between repetitions. Matlab 6.5 was used for management of acquisition parameters, storage, and processing of data relating to $\mathrm{COP}$ and to analyze the behavior of the stabilogram-diffusion graph of the COP through the random walk model established within this same program.

\section{Statistical analyses}

Data were presented using descriptive statistics (mean, standard deviation, percent, and rate). We also observed the skewness and kurtosis of the normality curve. The Shapiro-Wilk test indicated that the variables were not normally distributed. Therefore, the KruskalWallis and Mann-Whitney U tests were used for comparisons between groups. To compare the frequency of reporting of diseases and types of medication, the chi-squared test was used. Spearman's correlation was applied between the clinical variables (WOMAC score, pain intensity, severity of OA, history of falls, associated diseases, type of medication, dizziness, reported auditory and visual deficits, fear of falling measured by the FES-I score, physical activity, age, and weight) and the SDA parameters. For the analysis of the Spearman correlation between the variables. ${ }^{27}$ A significance level of 5\% $(\alpha=0.05)$ was adopted for all tests. The statistical package SPSS version 20.0 was used for the analyses.

\section{Results}

The groups were homogeneous with respect to age and height. The body weight and body mass index (BMI) of the osteoarthritis groups $\left(\mathrm{KOAG}^{\mathrm{F}}\right.$ and $\left.\mathrm{KOAG}^{\mathrm{NF}}\right)$ were significantly different to those of the $\mathrm{CG}$ (Table 1). The $\mathrm{KOAG}^{\mathrm{F}}$ and $\mathrm{KOAG}^{\mathrm{NF}}$ also reported a higher prevalence of musculoskeletal and/or connective tissue diseases than the CG. Among the most widely used drugs, the $\mathrm{KOAG}^{\mathrm{F}}$ and $\mathrm{KOAG}^{\mathrm{NF}}$ showed higher frequency of constant use of drugs for the musculoskeletal system than the $\mathrm{CG}$, and the $\mathrm{KOAG}^{\mathrm{F}}$ had a higher frequency of medication use for digestive and/or metabolic diseases than the $\mathrm{KOAG}^{\mathrm{NF}}$ (Table 2). In other clinical data, visual impairment, hearing impairment, and lack of dizziness were not significantly different between the groups. The reports of regular physical activity showed that the disabled subjects with KOA were more inactive than the asymptomatic ones, regardless of history of falls (Table 3 ).

\begin{tabular}{|c|c|c|c|c|}
\hline & KOAGF & KOAGNF & CG & \\
\hline Age (years) & $63.12( \pm 3.3)$ & $61.22( \pm 2.3)$ & $65.61( \pm 1.8)$ & NS \\
\hline Body weight (kg) & $80.45( \pm 3.2)$ & $83.13( \pm 3.3)$ & $65.47( \pm 2.9) * \Delta$ & $<0.001$ \\
\hline Height (m) & $1.57( \pm 0.02)$ & $1.62( \pm 0.02)$ & $1.50( \pm 0.08)$ & NS \\
\hline Body mass index (BMI) & $32.61( \pm 1.14)$ & $31.41( \pm 1.26)$ & $26.24( \pm I .0 I)^{* \Delta}$ & 0.001 \\
\hline
\end{tabular}

Abbreviations KOAGF, knee osteoarthritis group with history of falls; KOAG ${ }^{\mathrm{NF}}$, KOA group without history of falls;

*Significant differences between $\mathrm{KOAG}^{\mathrm{F}}$ and $\mathrm{CG}(\rho<0.05)$

$\Delta$ Significant differences between KOAGNF and CG $(\rho<0.05)$ 
Table 2 Health problems reported by groups

\begin{tabular}{|c|c|c|c|c|}
\hline & KOAGF\# & KOAGNF\# & CG & $\rho$ \\
\hline Carriers of Disease & 17 (94.4\%) & 17 (70.8\%) & $14(63.6 \%)$ & 0.068 \\
\hline \multicolumn{5}{|l|}{ Health Issues: } \\
\hline Nutritional and/or metabolic disorders & 7 (38.9\%) & $4(16.7 \%)$ & $3(13.6 \%)$ & 0.116 \\
\hline Nervous system diseases & I (5.6\%) & $3(12.5 \%)$ & $3(13.6 \%)$ & 0.684 \\
\hline Ear disorders & $3(16.7 \%)$ & $5(20.8 \%)$ & $2(9.1 \%)$ & 0.543 \\
\hline Circulatory system diseases & $10(55.6 \%)$ & II (45.8\%) & $8(36.4 \%)$ & 0.478 \\
\hline Digestive system diseases & $2(11.1 \%)$ & $2(8.3 \%)$ & $2(9.1 \%)$ & 0.953 \\
\hline Skin and/or subcutaneous tissue diseases & $2(11.1 \%)$ & $2(8.3 \%)$ & 0 & 0.306 \\
\hline $\begin{array}{l}\text { Musculoskeletal system and/or connective tissue } \\
\text { diseases }\end{array}$ & $15(83.3 \%)^{*}$ & $16(66.7 \%)^{*}$ & $3(13.6 \%)$ & $<0.001$ \\
\hline Genitourinary system diseases & $2(11.1 \%)$ & I (4.2\%) & I (4.5\%) & 0.603 \\
\hline Medicines & 14 (77.8\%) & $18(75 \%)$ & $13(59.1 \%)$ & 0.357 \\
\hline \multicolumn{5}{|l|}{ Medication use for: } \\
\hline Digestive and/or metabolic systems & $7(38.9 \%)^{*}$ & $2(8.3 \%)$ & $2(9.1 \%)$ & 0.016 \\
\hline Cardiovascular system & $9(50 \%)$ & 14 (58.3\%) & $9(40.9 \%)$ & 0.498 \\
\hline Musculoskeletal system & $10(55.5 \%)^{*}$ & $10(41.7 \%)^{*}$ & 0 & 0.001 \\
\hline Nervous system & $2(11.1 \%)$ & $3(12.5 \%)$ & $3(13.6 \%)$ & 0.972 \\
\hline Endocrine system & 0 & $5(20.8 \%)$ & $5(22.7 \%)$ & 0.097 \\
\hline Sensory organs & I (5.6\%) & 0 & I (4.5\%) & 0.738 \\
\hline Genitourinary system and sex hormones & 0 & 0 & I (4.5\%) & 0.379 \\
\hline
\end{tabular}

Abbreviations $\mathrm{KOAG}^{\mathrm{F}}$, Knee osteoarthritis group with history of falls; KOAG ${ }^{\mathrm{NF}}$, KOA group without history of falls;

*Significant difference compared to the control group.

\#Excluded KOA disease

Table 3 Clinical variables of the groups. data presented as mean (SD)

\begin{tabular}{|c|c|c|c|c|}
\hline & KOAGF & KOAGNF & CG & $\#$ \\
\hline Pain in the knee joint & $5.8( \pm 0.65)$ & $5.7( \pm 0.42)$ & - & 0.712 \\
\hline $\begin{array}{l}\text { Limitation in activities of daily living } \\
\text { (ADLs) }\end{array}$ & $14(77.8 \%)$ & 17 (70.8\%) & - & 0.443 \\
\hline WOMAC & $50.6 \mathrm{I}( \pm 5.67)^{*}$ & $44.91( \pm 5.42)^{*}$ & $98.3( \pm 2.2)$ & 0.479 \\
\hline FES & $41.50( \pm 4.4)^{*}$ & $31.23( \pm 4.3)^{*}$ & $21.20( \pm 1.03)$ & $<0.001$ \\
\hline MMSE & $26.94( \pm 0.47)$ & $28.30( \pm 0.32)$ & $27.62( \pm 0.44)$ & 0.66 \\
\hline Regular physical activity & $5(27.8 \%)$ & $9(37.5 \%)$ & 19 (86.4\%) & $<0.001$ \\
\hline Dizziness & 2 (II.1\%) & $5(20.8 \%)$ & I (4.5\%) & 0.243 \\
\hline Hearing deficit & I (5.6\%) & $3(12.5 \%)$ & $2(9.1 \%)$ & 0.935 \\
\hline Visual deficit & $10(55.6 \%)$ & $15(62.5 \%)$ & $15(68.2 \%)$ & 0.937 \\
\hline Fear of falling* & 17 (94.4\%) & 17 (70.8\%) & $10(45.5 \%)$ & 0.004 \\
\hline Average of any fall $<12$ months & $2.1( \pm 0.5)$ & - & - & - \\
\hline Restrictions after the fall & $6(33.33 \%)$ & - & - & - \\
\hline
\end{tabular}

Abbreviations KOAGF, Knee osteoarthritis group with history of falls; KOAG ${ }^{\mathrm{NF}}$, KOA group without history of falls \#Difference level between the KOAGF and KOAGNF

*Significant difference from the control group-CG, $\mathrm{p}<0.001$

Citation: Moreira ACSDS, Cardoso FL, Mazo GZ, et al. Influence of the clinical in postural balance in woman with knee osteoarthritis. Int Phys Med Rehab J. 2019;4(4):I35-143. DOI: 10.15406/ipmri.2019.04.00।88 
Differences in clinical data between individuals with knee osteoarthrosis

The assessment of the clinical status of the $\mathrm{KOAG}^{\mathrm{F}}$ showed grade 3 or 4 of joint cartilage damage in the right knee of $50 \%$ of the subjects and in the left knee of $66.6 \%$ of the subjects, respectively. The $\mathrm{KOAG}^{\mathrm{NF}}$ showed grade 3 or 4 in the right knee $(54.2 \%)$ and the left knee (58.4\%), respectively, in accordance with the KellgrenLawrence Grading Scale ${ }^{23}$. Pain intensity in the knee on the day of data collection, restriction to ADLs, and functional capacity and deficits showed no significant differences between the KOA groups. Most subjects with osteoarthritis $\left(\mathrm{KOAG}^{\mathrm{F}}=55.6 \%\right.$ and $\left.\mathrm{KOAG}^{\mathrm{NF}}=50 \%\right)$ had severe and very severe disability (Table 3 ).

Self-reported fear of falling was more prevalent in the subjects with osteoarthritis than in the asymptomatic subjects (CG), showing a significant difference $(\mathrm{p}<0.001)$. The $\mathrm{KOAG}^{\mathrm{F}}$ and $\mathrm{KOAG}^{\mathrm{NF}}$ groups had higher scores in the fear of falling scale (FES-I) than the CG $(p<0.001$ and $p=0.015$, respectively). The results showed no significant difference between the $\mathrm{KOAG}^{\mathrm{F}}$ and $\mathrm{KOAG}^{\mathrm{NF}}$. The $\mathrm{KOAG}^{\mathrm{F}}$ group reported a fall prevalence of $2.1( \pm 0.5)$ during the year, and $33.33 \%$ had restriction to ADLs after the fall. The analysis of the cognitive status showed no significant difference between groups (Table 3 ).

\section{Stabilogram behavior among individuals with knee osteoarthrosis}

The short-term (Dys) and long-term (Dyl) diffusion coefficients and the critical point coordinates $\left(\Delta \mathrm{x}^{2}\right)$ showed higher mean values in the subjects with osteoarthritis $\left(\mathrm{KOAG}^{\mathrm{F}}\right.$ and $\left.\mathrm{KOAG}^{\mathrm{NF}}\right)$ when compared to the CG subjects. Statistical differences were found in these variables in both visual conditions; however, in the mediolateral direction, there was no statistically significant difference between groups (Table 4).

Table 4 Comparison of stabilogram-diffusion parameters in the anteroposterior direction with eyes open and closed

\begin{tabular}{|c|c|c|c|c|}
\hline Variable & Groups & $\bar{X} \bar{x}$ & Sd & $\rho$ \\
\hline & $\mathrm{KOAG}^{\mathrm{F}}$ & 0.09 & \pm 0.04 & \\
\hline \multirow[t]{3}{*}{ Dyl AP EO $\left(\mathrm{mm}^{2} / \mathrm{s}\right)$} & KOAGNF & 0.09 & \pm 0.02 & 0.003 \\
\hline & $\mathrm{CG}^{\ddagger} \Delta$ & 0.02 & \pm 0.01 & \\
\hline & $\mathrm{KOAG}^{\mathrm{F}}$ & 0.31 & \pm 0.08 & \\
\hline \multirow[t]{3}{*}{ Dys AP EO $\left(\mathrm{mm}^{2} / \mathrm{s}\right)$} & $\mathrm{KOAG}^{\mathrm{NF}}$ & 0.29 & \pm 0.04 & 0.006 \\
\hline & $\mathrm{CG}^{\ddagger} \Delta$ & 0.19 & \pm 0.08 & \\
\hline & $\mathrm{KOAG}^{\mathrm{F}}$ & 0.25 & \pm 0.03 & \\
\hline \multirow[t]{3}{*}{ HLT AP EO (adc) } & KOAGNF & 0.27 & \pm 0.03 & 0.987 \\
\hline & CG & 0.25 & \pm 0.03 & \\
\hline & $\mathrm{KOAG}^{\mathrm{F}}$ & 0.26 & \pm 0.04 & \\
\hline \multirow[t]{3}{*}{ HST EO AP } & $\mathrm{KOAG}^{\mathrm{NF}}$ & 0.26 & \pm 0.04 & 0.396 \\
\hline & CG & 0.31 & \pm 0.03 & \\
\hline & $\mathrm{KOAG}^{\mathrm{F}}$ & 0.51 & \pm 0.10 & \\
\hline \multirow[t]{3}{*}{$\Delta \mathrm{x}^{2}$ AP EO $\left(\mathrm{mm}^{2}\right)$} & $\mathrm{KOAG}^{\mathrm{NF}}$ & 0.51 & \pm 0.08 & 0.004 \\
\hline & $\mathrm{CG}^{\ddagger} \Delta$ & 0.25 & \pm 0.08 & \\
\hline & $\mathrm{KOAG}^{\mathrm{F}}$ & 0.94 & \pm 0.19 & \\
\hline \multirow[t]{3}{*}{$\Delta r^{2} c$ AP EO (s) } & $\mathrm{KOAG}^{\mathrm{NF}}$ & 0.83 & \pm 0.12 & 0.658 \\
\hline & CG & 0.92 & \pm 0.14 & \\
\hline & KOAG $^{F}$ & 0.07 & \pm 0.03 & \\
\hline \multirow[t]{3}{*}{ Dyl AP EC $\left(\mathrm{mm}^{2} / \mathrm{s}\right)$} & $\mathrm{KOAG}^{\mathrm{NF}}$ & 0.08 & \pm 0.02 & 0.001 \\
\hline & $\mathrm{CG}^{\ddagger} \boldsymbol{\Lambda}$ & 0.01 & \pm 0.01 & \\
\hline & $\mathrm{KOAG}^{\mathrm{F}}$ & 0.48 & \pm 0.13 & \\
\hline \multirow[t]{3}{*}{ Dys AP EC $\left(\mathrm{mm}^{2} / \mathrm{s}\right)$} & KOAG ${ }^{N F}$ & 0.59 & \pm 0.01 & 0.004 \\
\hline & $\mathrm{CG}^{\ddagger} \boldsymbol{\Lambda}$ & 0.22 & \pm 0.06 & \\
\hline & KOAGF $^{F}$ & 0.16 & \pm 0.02 & \\
\hline \multirow[t]{3}{*}{ HLT AP EC } & KOAG ${ }^{\mathrm{NF}}$ & 0.19 & \pm 0.02 & 0.664 \\
\hline & CG & 0.18 & \pm 0.03 & \\
\hline & KOAGF & 0.33 & \pm 0.04 & \\
\hline \multirow[t]{2}{*}{ HST AP EC } & KOAGNF & 0.33 & \pm 0.03 & 0.693 \\
\hline & CG & 0.36 & \pm 0.03 & \\
\hline
\end{tabular}


Table Continued

\begin{tabular}{|c|c|c|c|c|}
\hline Variable & Groups & $\bar{X} \bar{x}$ & Sd & $\rho$ \\
\hline \multirow{3}{*}{$\Delta \mathrm{x}^{2} \mathrm{AP}$ EC $\left(\mathrm{mm}^{2}\right)$} & $\mathrm{KOAG}^{\mathrm{F}}$ & 1.30 & \pm 0.52 & \multirow{3}{*}{0.007} \\
\hline & KOAGNF & 1.03 & \pm 0.02 & \\
\hline & $\mathrm{CG}^{\ddagger} \boldsymbol{\Delta}$ & 0.44 & \pm 0.16 & \\
\hline \multirow{3}{*}{$\Delta r^{2} c A P E C(s)$} & $\mathrm{KOAG}^{\mathrm{F}}$ & 0.84 & \pm 0.16 & \multirow{3}{*}{0.908} \\
\hline & KOAGNF & 0.96 & \pm 0.17 & \\
\hline & CG & 0.86 & \pm 0.13 & \\
\hline
\end{tabular}

Abbreviations KOAGF, group KOA with fall history; KOAGNF, group KOA without fall history; CG, control group; EO, eyes open; EC, eyes closed; Dys, short interval of time; Dyl, long interval; HLT, hurst exponent over time; HST, Hurst exponent in a short period of time; $\Delta \mathrm{x}^{2}$, position of the critical point; $\Delta \mathrm{r}^{2} \mathrm{c}$, moment of critical point. $\rho$, Significance level of the one-way ANOVA test.

‡ Significant difference between KOAG ${ }^{\mathrm{F}}$ and CG $(\rho<0.05)$

$\Delta$ Significant difference between KOAGNF and CG $(\rho<0.05)$

\section{Association between the clinical data and the stabilogram diffusion analysis}

The results showed that the variables related to clinical data (age, body mass, physical activity, fear of falling (FES-I), functional capacity (WOMAC), severity of OA, pain intensity, and the presence of musculoskeletal diseases) had a moderate to strong correlation with the SDA parameters (Dys, Dyl, and $\Delta \mathrm{x}^{2}$ ) in both visual conditions in the AP direction. The use of medication for the musculoskeletal system showed moderate correlation with Dyl in both visual conditions in the AP direction. There were no significant correlations between BMI, visual impairment, hearing impairment, reports of dizziness, and history of falls outside the SDA parameters (Table 5).

Table 5 - Correlation between the clinical/social aspects and the stabilogram-diffusion parameters in the anteroposterior direction with eyes open and closed including all subjects.

\begin{tabular}{|c|c|c|c|c|c|c|c|c|c|c|c|c|c|}
\hline \multirow{2}{*}{ Variables } & & Dys & Dyl & HST & HLT & $\Delta \mathrm{x}^{2}$ & $\Delta r^{2} c$ & Dys & Dyl & HST & HLT & $\Delta \mathrm{x}^{2}$ & $\Delta r^{2} c$ \\
\hline & & $A P \_E O$ & AP_EO & _AP_EO & AP_EO & AP_EO & AP_EO & AP_EC & $A P_{-} E C$ & $A P_{-} E C$ & $A P_{-} E C$ & $A P \_E C$ & $A P_{-} E C$ \\
\hline \multirow{2}{*}{ Age } & $r$ & $0.464 * *$ & $0.559 * *$ & 0.215 & 0.033 & $0.416^{* *}$ & 0.096 & $0.48 I^{* *}$ & $0.522 * *$ & 0.043 & 0.082 & $0.487 * *$ & 0.081 \\
\hline & $\rho$ & $<0.001$ & $<0.001$ & 0.100 & 0.803 & $<0.001$ & 0.467 & $<0.001$ & $<0.001$ & 0.743 & 0.535 & $<0.001$ & 0.540 \\
\hline \multirow{2}{*}{ Body Weight } & $r$ & $0.488^{* * k}$ & $0.393^{* *}$ & 0.061 & 0.147 & $0.409^{* *}$ & 0.127 & $0.527^{* k}$ & $0.367^{* *}$ & 0.076 & 0.222 & $0.493^{* *}$ & 0.058 \\
\hline & $\rho$ & $<0.001$ & 0.002 & 0.646 & 0.267 & 0.001 & 0.339 & $<0.001$ & 0.004 & 0.566 & 0.091 & $<0.001$ & 0.663 \\
\hline \multirow{2}{*}{ Physical activity } & $r$ & $0.384^{* *}$ & $0.330^{*}$ & 0.228 & 0.123 & $0.403^{* *}$ & 0.006 & $0.380^{* *}$ & $0.454^{* *}$ & 0.233 & 0.022 & $0.425^{* *}$ & 0.115 \\
\hline & $\rho$ & 0.002 & 0.010 & 0.091 & 0.350 & 0.001 & 0.967 & 0.003 & 0.000 & 0.073 & 0.866 & 0.001 & 0.383 \\
\hline \multirow{2}{*}{$\begin{array}{l}\text { Severity of } \\
\text { Osteoarthritis }\end{array}$} & $r$ & $0.487^{* * *}$ & $0.497^{* *}$ & 0.179 & 0.129 & $0.522^{* *}$ & 0.026 & $0.428^{* *}$ & $0.505^{* *}$ & 0.151 & 0.077 & $0.532^{* *}$ & 0.038 \\
\hline & $\rho$ & $<0.001$ & $<0.001$ & 0.171 & 0.327 & $<0.001$ & 0.843 & $<0.001$ & $<0.001$ & 0.250 & 0.559 & $<0.001$ & 0.774 \\
\hline \multirow{2}{*}{ VAS for pain } & $r$ & $0.361^{* *}$ & $0.338^{* * *}$ & 0.064 & 0.033 & $0.342^{* *}$ & 0.043 & $0.433^{* *}$ & $0.502^{* *}$ & 0.077 & 0.076 & $0.438^{* *}$ & 0.079 \\
\hline & $\rho$ & 0.005 & 0.008 & 0.627 & 0.802 & 0.007 & 0.746 & 0.001 & $<0.001$ & 0.556 & 0.565 & $<0.001$ & 0.548 \\
\hline \multirow{2}{*}{$\begin{array}{l}\text { Musculoskeletal } \\
\text { system disease }\end{array}$} & $r$ & $0.447^{* *}$ & $0.437^{* * *}$ & 0.183 & 0.013 & $0.439^{* *}$ & 0.016 & $0.473^{* *}$ & $0.590^{* * *}$ & 0.154 & 0.013 & $0.493^{* *}$ & 0.039 \\
\hline & $\rho$ & $<0.001$ & $<0.001$ & 0.163 & 0.921 & 0.000 & 0.903 & $<0.001$ & $<0.001$ & 0.239 & 0.921 & $<0.001$ & 0.770 \\
\hline \multirow{2}{*}{$\begin{array}{l}\text { Medication for } \\
\text { the Muscular } \\
\text { System }\end{array}$} & $r$ & 0.221 & $0.35 I^{\text {*** }}$ & 0.061 & 0.078 & 0.252 & 0.074 & 0.271 & $0.381 *$ & 0.132 & 0.221 & 0.272 & 0.147 \\
\hline & $\rho$ & 0.090 & 0.006 & 0.644 & 0.555 & 0.052 & 0.572 & 0.072 & 0.003 & 0.175 & 0.082 & 0.071 & 0.186 \\
\hline \multirow{2}{*}{$\begin{array}{l}\text { Falls in the last } \\
\text { year }\end{array}$} & $r$ & $0.337 * *$ & $0.354 * *$ & 0.147 & 0.028 & $0.364 * *$ & 0.032 & $0.348 * *$ & $0.448 * *$ & 0.097 & 0.046 & $0.389 * *$ & 0.035 \\
\hline & $\rho$ & 0.008 & 0.006 & 0.261 & 0.830 & 0.004 & 0.807 & 0.006 & $<0.001$ & 0.463 & 0.729 & 0.002 & 0.791 \\
\hline \multirow{2}{*}{ FES-I } & $r$ & $0.472^{*}$ & $0.478^{*}$ & 0.040 & 0.178 & $0.469^{*}$ & 0.006 & $0.313^{*}$ & $0.306^{*}$ & 0.009 & 0.035 & $0.346^{*}$ & 0.105 \\
\hline & $\rho$ & $<0.001$ & $<0.001$ & 0.797 & 0.247 & 0.000 & 0.970 & 0.039 & 0.043 & 0.955 & 0.819 & 0.021 & 0.496 \\
\hline \multirow{2}{*}{ WOMAC } & $r$ & $0.420^{* *}$ & $0.445^{* *}$ & 0.198 & 0.041 & $0.413^{* *}$ & 0.050 & $0.455^{* *}$ & $0.493^{* k}$ & 0.164 & 0.192 & $0.46 I^{* *}$ & 0.098 \\
\hline & $\rho$ & $<0.001$ & $<0.001$ & 0.130 & 0.754 & 0.001 & 0.705 & $<0.001$ & $<0.001$ & 0.211 & 0.142 & $<0.001$ & 0.458 \\
\hline
\end{tabular}

Legend: EO - eyes open; EC - eyes closed; Dys - short-term interval; Dyl - long-term interval; HLT - Hurst exponent over time; HST - Hurst exponent in a short period of time; $\Delta x^{2}$ - position of the critical point; $\Delta r^{2} c$ - moment of critical point; $\rho$ - significance level of the Spearman test; $r$ - correlation level of the Spearman test. 


\section{Discussion}

\section{Behavior of the stabilogram-diffusion curve}

The Dys and Dyl values of the subjects with KOA were higher than in the asymptomatic group, showing a deficit in sensory detection and proprioceptive feedback that negatively affected body balance. The increase in AP activity in the subjects with KOA could reflect an attempt to keep the movements in a stable ML direction. ${ }^{28,29}$ The subjects with KOA used offsets and adjustments in the AP direction through two mechanisms of postural control, i.e., open-loop (open circuit) and closed-loop (closed circuit). This is a compensatory strategy that aims to neutralize the effects of restricted motion in the ML direction. ${ }^{28,29}$

Some authors ${ }^{29,30}$ suggest that the short-term region (Dys) corresponds to the open circuit mechanism that does not allow direct control by the central nervous system. Therefore, posture stability is only dependent on the rigidity (stiffness) of the muscle. ${ }^{3,8}$ The subjects with KOA have higher Dys values, which, according to Laughton et $\mathrm{al}^{31}{ }^{31}$ represent a delay in transmission of sensory-processing and detection of muscle activation. Such changes can occur in individuals under conditions of low muscular co-activation or with a decline in nerve conduction speed, ${ }^{31}$ both situations present in the pathophysiology of KOA. ${ }^{5,15,17,32}$ Thus, increased muscle activity may be responsible for the increased stochastic activity (Dys) and hence may compromise an individual's ability to maintain postural stability, increasing the risk of falling. ${ }^{3,5,8}$

The subjects with KOA had higher Dyl when compared with the asymptomatic ones. The region of Dyl corresponds to the mechanism in a closed circuit, which utilizes sensory information to generate corrective postural disturbances to compensate for muscle torque. ${ }^{30,31}$ In this sense, it explains the increased reflex time, decreased proprioception, and muscular weakness reported as common consequences of the aging process and the KOA pathophysiology. Therefore, the feedback mechanisms of a closed system present a delay in activation. ${ }^{28}$

In relation to the history of falls, this study found no significant difference between the Dys and Dyl of the $\mathrm{KOAG}^{\mathrm{F}}$ and $\mathrm{KOAG}^{\mathrm{NF}}$, perhaps because they were homogeneous in terms of clinical data. Therefore, regardless of history of falls, both groups have deficits in sensory detection and proprioceptive feedback, negatively affecting body balance and contributing to increased risk of falling. Although this study did not evaluate these variables, it is possible that environmental factors can be predictors of falls in this population.

In relation to the intermediate phase between the short- and longterm ranges, this study found a significant difference in the value of $\Delta \mathrm{x}^{2}$ between the subjects with KOA and the asymptomatic subjects, perhaps explained by the higher Dys. It is possible that the KOA groups used a larger area of their support base and had greater sway than did the asymptomatic subjects. Similarly, Tanaka et al. ${ }^{33}$ reported higher values of $\Delta \mathrm{x}^{2}$ in young adults with poorer ability to maintain one-leg balance, suggesting that the group at high risk of falling used most of its support base area more quickly than the low-risk group.

\section{Correlation between clinical parameters and the stabilogram diffusion analysis}

The Dys, Dyl, and $\Delta \mathrm{x}^{2}$ parameters increased with age as confirmed by previous studies ${ }^{31,34,35}$ that analyzed the effect of the aging process on the stabilogram-diffusion curve. These authors suggest that advancing age increases the postural instability in these parameters, a fact confirmed in this study.

When analyzing the clinical data of the subjects, it was noted that BMI does not show any correlation with the SDA, however the increase in body mass enabled these subjects to maintain postural stability, indicating that the increase in body mass is a factor of greater influence than BMI for postural instability. Hue et al. ${ }^{36}$ state that the correlation between body mass and postural stability is a result of assuming an upright posture, a position in which the human body acts as an inverted pendulum rotating around the ankle joint. In this context, the center of mass is located near the anterior edge of the support base, due to the increase in body mass in an attempt to maintain balance on the basis that the ankle support will increase its torque, and therefore postural sway. Thus, previous studies ${ }^{36,37}$ conclude that body mass is a predictor of postural instability, which is an essential prerequisite for increased risk of falls in ADLs.

Increased body mass is considered one of the etiological factors responsible for the development of musculoskeletal diseases. ${ }^{2,38}$ In this context, the results indicated that the greater prevalence of disease increased musculoskeletal Dys, Dyl, and $\Delta \mathrm{x}^{2}$, suggesting that the presence of KOA indicates postural instability. Consequently, when analyzing the medications regularly used by the study subjects, we noticed a higher prevalence of drugs for musculoskeletal disorders, i.e., anti-inflammatories, analgesics, and muscle relaxants, which correlated with increased Dyl, meaning a time delay in muscle activation to maintain postural stability. ${ }^{28}$ Studies indicate that chronic diseases and drug use are risk factors for falls and functional dependence..$^{39,40}$

These associations, reinforced by a clear dose-response gradient, can reveal two mechanisms, one for etiological factors and the other a reverse causality, since individuals with KOA tend to be more inactive and therefore have higher body mass index. ${ }^{38}$ Thus, the results showed that the worsening in KOA leads to greater postural instability. More specifically, the greater the joint cartilage damage, functional disability, physical inactivity, and pain intensity are, the greater the changes in Dys, Dyl, and $\Delta \mathrm{x}^{2}$ values. The increased fear of falling (FES-I) also correlated with these parameters, indicating that this fear fosters insecurity, dependence in ADLs, restriction to social activities, and therefore decreased autonomy over time.

According to Yahia et al. ${ }^{15}$ deficits in balance and functional capacity may be related to a reduction in motor neuron excitability of the quadriceps muscle and subsequent loss of strength and proprioception. Mohammadi et al. ${ }^{9}$ found that women with KOA showed reduced equilibrium due to impaired knee joint proprioception and quadriceps strength. Other authors ${ }^{9,12}$ also associated balance deficits with decreased proprioception and lack of confidence regarding the performance of ADLs. Similar findings in the present study allow us to conclude that the higher the level of postural instability, the higher the risks of suffering from fear of falling during ADLs.

In this context, some authors ${ }^{3,8,32}$ reported that patients with KOA exhibit deficits in the control of knee joint stability, which are reflected in the Dys, Dyl and, $\Delta \mathrm{x}^{2}$. This increase in stochastic activity of the $\mathrm{COP}$ and the delay in sensory detection and proper postural control to stabilize postural sway responses can cause a decrease in confidence regarding performance of ADLs and a tendency to be more inactive, thereby undermining the mechanisms of motor control stabilization of body balance and favoring the occurrence of falls in this population. 
Nevertheless, this finding was not observed in previous studies ${ }^{6,9,41,42}$ that used the traditional posturographic analysis of COP. Therefore, we believe that SDA is more sensitive in detecting the relationship between the predictors that can alter postural stability and clinical parameters.

This study had some significant limitations, such as a small number of subjects, which prevented inference about the power of each independent variable on the postural balance of women with KOA. We suggest that future studies include a sample size large enough to determine the power of each independent variable to predict postural instability in these subjects and combine that with electromyography to ascertain the level of muscle activation, evaluation of proprioception, and muscle strength.

\section{Conclusion}

When the SDA variables were compared between the groups, the subjects with $\mathrm{KOA}\left(\mathrm{KOAG}^{\mathrm{F}}\right.$ and $\left.\mathrm{KOAG}^{\mathrm{NF}}\right)$, both with eyes open and closed, showed higher values for short-term (Dys) and long-term (Dyl) diffusion coefficients and critical point coordinates $\left(\Delta x^{2}\right)$ in the AP direction than the asymptomatic subjects. These findings suggest that the KOA groups require a longer time for sensory detection in order to process neural and sensory transmission and muscle activation. In addition, the subjects with KOA had higher stochastic activity of the COP, as evidenced by $\Delta \mathrm{x}^{2}$, and thus required more time to acquire postural stability and used a larger area of their support base in the COP displacement than the subjects in the control group.

Postural instability was associated with clinical and social aspects of balance, including greater body mass, higher level of inactivity, greater severity of osteoarthritis, worse pain symptoms, chronic musculoskeletal diseases, use of medication for the musculoskeletal system, worse functional capacity, and greater fear of falling. Therefore, we conclude that the clinical and social context, rather than history of falls, is an important predictor for the risk of falling.

\section{Acknowledgments}

None.

\section{Conflicts of interest}

The authors declare no conflicts of interest.

\section{References}

1. Ganasegeran K, Menke JM, Ramaswamy VM, et al. Level and determinants of knowledge of symptomatic knee osteoarthritis among railway workers in Malaysia. Biomed Res Int. 2014;370273.

2. Jhun HJ, Sung NJ, Kim SY. Knee pain and its severity in elderly Koreans: prevalence risk factors and impact on quality of life. J Korean Med Sci. 2013;28:1807-1813.

3. Kim I, Kim HA, Seo YI, et al. The prevalence of knee osteoarthritis in elderly community residents in Korea. $J$ Korean Med Sci. 2010;25(2):293-298.

4. Michael JW, Schlüter-Brust KU, Eysel P. The epidemiology, etiology, diagnosis, and treatment of osteoarthritis of the knee. Dtsch Arztebl Int. 2010;107(9):152-162.
5. Kim D, Park G, Kuo LT2, Park W. The effects of pain on quadriceps strength, joint proprioception and dynamic balance among women aged 65 to 75 years with knee osteoarthritis. BMC Geriatr. 2018;9(8):774 780.

6. Taglietti M, Dela Bela LF, Dias JM, et al. Postural sway, balance confidence, and fear of falling in women with knee osteoarthritis in comparison to matched controls. PMR. 2018;18(1):245.

7. Takacs J, Carpenter MG, Garland SJ, et al. The role of neuromuscular changes in aging and knee osteoarthritis on dynamic postural control. Aging Dis. 2013;4:84-99.

8. Zeni JA, Higginson JS. Knee osteoarthritis affects the distribution of joint moments during gait. Knee. 2011;18:156-159.

9. Mahmoudian A, Van Dieen JH., Baert IA., et al. Changes in proprioceptive weighting during quiet standing in women with early and established knee osteoarthritis compared to healthy controls. Gait Posture. 2016;44:184-188.

10. Shih-Hung C, Mao-Hsiung H, Tien-Wen C, et al. Effect of knee sleeve on static and dynamic balance in patients with knee osteoarthritis Kaohsiung. J Med Sci. 2007;23:405-411.

11. Parveen H, Noohu MM. Evaluation of psychometric properties of Tinetti performance-oriented mobility assessment scale in subjects with knee osteoarthritis. Hong Kong Physiother J. 2017;36:25-32.

12. Alencar MA, Arantes PMM, Dias JMD, et al. Muscular function and functional mobility of faller and non-faller elderly women with osteoarthritis of the knee. Braz J Med Biol Res. 2007;40:277-283.

13. Hurley MV, Scott DL, Rees J, et al. Sensorimotor changes and functional performance in patients with knee osteoarthritis. Ann Rheum Dis. 1997;56:641-648.

14. Levinger P, Nagano H, Downie C., et al. Biomechanical balance response during induced falls under dual task conditions in people with knee osteoarthritis. Gait Posture. 2016;48:106-112.

15. Yahia A, Ksentini H, Jribi S, et al. Correlations between isokinetic knee muscle strength, postural and functional parameters in knee osteoarthritis patients. Ann Phys Rehabil Med. 2016;59S:e156.

16. Sørensen RR, Jørgensen MG, Rasmussen S, et al. Impaired postural balance in the morning in patients with knee osteoarthritis. Gait Posture. 2014;39(4):1040-1044.

17. Pua YH, Liang Z, Ong PH, et al. Associations of knee extensor strength and standing balance with physical function in knee osteoarthritis. Arthritis Care Res (Hoboken). 2011;63:1706-1714.

18. Tarigan TJ, Kasjmir YI, Atmakusuma D, et al. The degree of radiographic abnormalities and postural instability in patients with knee osteoarthritis. Acta Med Indones. 2009;41:15-19.

19. Tuna S, Balci N. The relationship between radiological severity and functional status in patients with knee osteoarthritis. Clin Rheumatol. 2014;33(5):667-670.

20. Turcot K, Hagemeister N, De Guise JA, et al. Evaluation of unipodal stance in knee osteoarthritis patients using knee accelerations and center of pressure. Osteoarthritis Cartilage. 2009;19:281-286.

21. Russell M, Hill K, Day L, et al. Predictors of long-term function in older community-dwelling people who have presented to an emergency department after a fall: A cohort study. Australas $J$ Ageing. 2015;34(1):47-52. 
22. Roos PE, Dingwell JB. Using dynamic walking models to identify factors that contribute to increased risk of falling in older adults. Hum Mov Sci. 2013;32:984-996.

23. Toda Y, Segal N. Usefulness of an insole with subtalar strapping for analgesia in patients with medial compartment osteoarthritis of the knee. Arthr Rheum. 2002;47(5):468-473.

24. Folstein MF, Folstein SE, Mchugh PR. Mini-mental state"- A practical method for grading the cognitive state of patients for the clinician. $J$ Psychiat Res. 1975;12:189-198.

25. Camargo FFO, Dias RC, Dias JMD, et al. Cross-cultural adaptation and evaluation of the psychometric properties of the Falls efficacy scale international among elderly Brazilians (FES-I-BRAZIL). Rev Bras Fisioter. 2010;14(3):237-243.

26. Collins JJ, De Luca CJ. Open loop and closed-loop control of posture: a random-walk analysis of center of pressure trajectories. Exp Brain Res. 1993;95:308-313

27. Cohen J. Statistical power analysis for the behavioral sciences. 2nd ed. Hillsdale: Lawrence Erbaum; 1988.

28. Kurz I, Oddsson L, Melzer I. Characteristics of balance control in older persons who fall with injury-A prospective study. J Electromyo Kinesio. 2013;23:814-819.

29. Mitchell SL, Collins JJ, De Luca CJ, et al. Open-loop and closedloop postural control mechanisms in Parkinson's disease: increased mediolateral activity during quiet standing. Neurosci Lett. 1995;197(2):133-136.

30. Collins JJ, De Luca CJ. Random walking during quiet standing. Physical Phys Rev Lett. 1994;73(5):764-767.

31. Laughton CA, Slavin M, Katdare K, et al. Aging muscle activity and balance control: physiologic changes associated with balance impairment. Gait Posture. 2003;18:101-108.

32. Shakoor N, Lee KJ, Fogg LF, et al. The relationship of vibratory perception to dynamic joint loading radiographic severity and pain in knee osteoarthritis. Arthritis Rheum. 2012;64:181-186.
33. Tanaka H, Uetake T, Kuriki S, et al. Changes in centro-pressure dynamics during upright standing related to decreased balance control in young adults: fractional Brownian motion analysis. J Hum Ergol. 2002;31(12):1-11.

34. Lervik J, Ledin T. Analysis of differences in postural control between elderly and young healthy subjects using chaos theory. Acta Orl. 2007;25(2):141-146.

35. Norris JA, Marsh AP, Smith IJ, et al. Ability of static and statistical mechanics posturographic measures to distinguish between age and fall risk. J Biomech. 2005;38:1263-1272.

36. Hue $\mathrm{O}$, Simoneau M, Marcotte $\mathrm{J}$, et al. Body weight is a strong predictor of postural stability. Gait Posture. 2007;26:32-38.

37. Takacs J, Carpenter MG, Garland SJ, et al. Factors associated with dynamic balance in people with knee osteoarthritis. Arch Phys Med Rehabil. 2015;96(10):1873-1879.

38. Dunlop DD, Song J, Semanik PA, et al. Physical activity levels and functional performance in the osteoarthritis initiative: a graded relationship. Arthritis Rheum. 2011;63(1):127-136.

39. Lawlor DA, Patel R, Ebrahim S. Association between falls in elderly women and chronic diseases and drug use: cross sectional study. $B M J$. 2003;327(7417):712-717.

40. Rubenstein LZ, Josephson KR. The epidemiology of falls and syncope. Clin Geriatr Med. 2002;18(2):141-158.

41. Hassan BS, Mockett S, Doherty M. Static postural sway, proprioception, and maximal voluntary quadriceps contraction in patients with knee osteoarthritis and normal control subjects. Ann Rheum Dis. 2001;60(6):612-618.

42. Hinman RS, Bennell KL, Metcalf BR, et al. Balance impairments in individuals with symptomatic knee osteoarthritis: a comparison with matched controls using clinical tests. Rheumatology. 2002;41(12):13881394. 